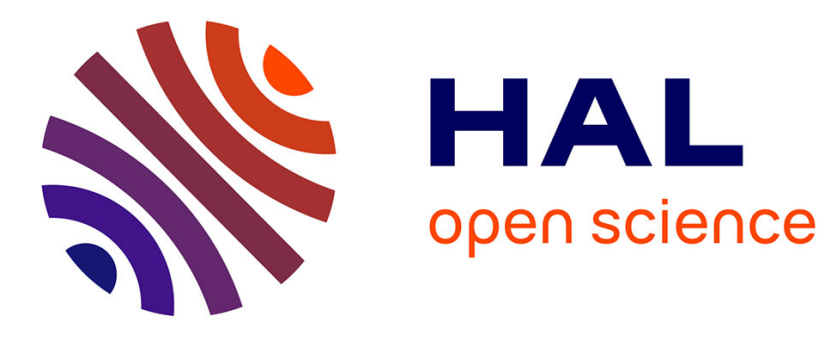

\title{
Optimal disturbances in swept Hiemenz flow
}

Alan Guégan, Patrick Huerre, Peter J. Schmid

\section{To cite this version:}

Alan Guégan, Patrick Huerre, Peter J. Schmid. Optimal disturbances in swept Hiemenz flow. Journal of Fluid Mechanics, 2007, 578 (may), pp.223-232. 10.1017/s0022112007005162 . hal-01023334

\section{HAL Id: hal-01023334 \\ https://hal-polytechnique.archives-ouvertes.fr/hal-01023334}

Submitted on 20 Jul 2014

HAL is a multi-disciplinary open access archive for the deposit and dissemination of scientific research documents, whether they are published or not. The documents may come from teaching and research institutions in France or abroad, or from public or private research centers.
L'archive ouverte pluridisciplinaire HAL, est destinée au dépôt et à la diffusion de documents scientifiques de niveau recherche, publiés ou non, émanant des établissements d'enseignement et de recherche français ou étrangers, des laboratoires publics ou privés. 


\title{
Optimal disturbances in swept Hiemenz flow
}

\author{
ALAN GUÉGAN, PATRICK HUERRE \\ AND PETER J. SCHMID \\ Laboratoire d'Hydrodynamique (LadHyX), CNRS-École Polytechnique, \\ F-91128 Palaiseau, France
}

(Received 4 December 2006 and in revised form 24 January 2007)

The initial perturbation with the largest transient energy growth is computed in the context of the swept leading-edge boundary layer. The highest energy amplification is found for perturbations which are homogeneous in the spanwise $z$-direction, although on shorter time scales the most amplified disturbances have a finite spanwise wavenumber. In both cases the production term associated with the shear of the spanwise velocity is responsible for the energy amplification in the perturbation energy equation. A connection is made with the amplification mechanism exhibited by optimal perturbations in streaky boundary layers (Hoepffner et al. J. Fluid Mech. vol. 537, 2005, p. 91) and the results are compared to the optimal Görtler-Hämmerlin disturbances computed by Guégan et al. (J. Fluid Mech. vol. 566, 2006, p. 11).

\section{Introduction}

Linear stability analysis falls short of explaining the mechanisms that lead to turbulence because it focuses only on the long-term behaviour of infinitesimal disturbances, whereas short-term phenomena may be crucial to transition. The interest in transient growth has been boosted by the possibility of computing the initial perturbation of a given shear flow that is most amplified over a finite time span, referred to as the optimal perturbation. It has been shown in several prototypical flows that the energy of initially infinitesimal disturbances may be amplified by several orders of magnitude, reaching levels at which nonlinear effects become significant.

The boundary layer at the leading edge of swept wings sketched in figure 1 is one among many examples of shear flows that can sustain transient energy amplification, such as Couette, Poiseuille and Blasius velocity profiles (Butler \& Farrell 1992). Guégan, Schmid \& Hnerre (2006) have demonstrated that the energy of a particular class of perturbations with a spatial structure that satisfies the so-called GörtlerHämmerlin assumption may be amplified by up to three orders of magnitude over finite time.

The strength of the sweep velocity $W$ in the spanwise $z$-direction is a crucial parameter for perturbation growth. In commercial airplanes with a sweep angle close to $30^{\circ}$, the vicinity of the attachment line is characterized by strong advection in the spanwise direction, which may therefore be referred to as the streamwise direction. The associated Reynolds number based on the sweep velocity lies around $R e=10^{3}$. In two-dimensional (Butler \& Farrell 1992) and three-dimensional (Corbett \& Bottaro 2001) boundary layers the optimal perturbations take the shape of vortical structures aligned with the mean streamwise advection and periodically distributed along the wall, perpendicular to the main stream. The so-called lift-up mechanism (Landahl 1980) is responsible for the energy amplification of such disturbances. 


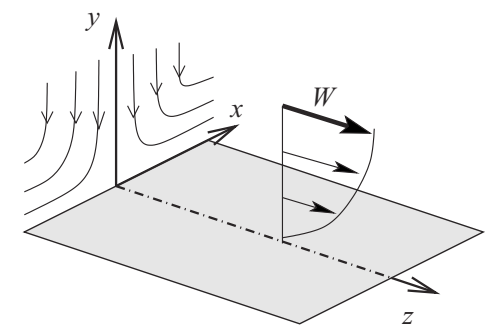

FIGURE 1. Sketch of swept Hiemenz flow showing the dividing streamlines in the chordwise $x$-direction and the sweep velocity $W$ in the spanwise $z$-direction.

The restrictive Görtler-Hämmerlin assumption, however, cannot describe streamwise vortices periodically distributed in the chordwise $x$-direction, since this assumption requires the chordwise $u$-velocity component of the perturbations to grow linearly away from the attachment line. It can be suspected that on relaxing the GörtlerHämmerlin hypothesis one may expect stronger energy growth than has been found by Guégan et al. (2006).

In the present study, direct numerical simulations have been used in conjunction with a gradient optimization algorithm in order to determine the general threedimensional optimal perturbation in swept Hiemenz flow. The dependence of the energy growth on the Reynolds number and the spanwise wavenumber has been investigated and the spatial structure of the optimal perturbation has been determined. The results are discussed in light of the previous studies of Hoepffner, Brandt \& Henningson (2005) on optimal perturbations of nonlinearly saturated streamwise streaks and of Guégan et al. (2006) on optimal perturbations within the GörtlerHämmerlin framework.

\section{Flow configuration and numerical techniques}

\subsection{Linear perturbations in swept Hiemenz flow}

Swept Hiemenz flow is an exact solution of the Navier-Stokes equations. It models the flow near the leading edge of a swept wing in the neighbourhood of the attachment line, on each side of which the impinging flow divides symmetrically (figure 1). The stretching chordwise velocity $U(x, y)$ is assumed to increase linearly with the chordwise $x$-direction, whereas the wall-normal velocity $V(y)$ and the spanwise sweep velocity $W(y)$ are assumed to be homogeneous in $x$. The only flow parameter is the Reynolds number

$$
R e=\frac{W_{\infty}(v / S)^{1 / 2}}{v}
$$

based on the stretching rate $S=(\mathrm{d} U / \mathrm{d} x)_{y \rightarrow \infty}$, the sweep velocity at infinity $W_{\infty}$ and the kinematic viscosity $v$. The Reynolds number quantifies the sweep angle and vanishes when the leading edge is perpendicular to the impinging flow.

Infinitesimal perturbations $(u, v, w, p)$ periodic in the spanwise $z$-direction with a spanwise wavenumber $k$ are superimposed on this steady base flow. The perturbation energy is taken to be

$$
E=\frac{1}{2} \int_{y>0} \lambda_{E}(x)\left(u^{2}+v^{2}+w^{2}\right) \mathrm{d} x \mathrm{~d} y \mathrm{~d} z
$$


where $\lambda_{E}(x)$ is a weighting function. The reason for introducing $\lambda_{E}$ is that swept Hiemenz flow is a degenerate model that does not take into account the leading-edge curvature. In particular the chordwise velocity $U$ increases linearly away from the attachment line. The usual way to avoid this singularity is to include a Gaussian energy weight in $x$ as in Obrist \& Schmid (2003a). In addition to focusing on the perturbations of most interest which are located close to the attachment line, a Gaussian weight function is justified mathematically in that it defines a proper scalar product for Hermite polynomial decompositions in $x$, thereby allowing the comprehensive analysis of the stability equations (Obrist \& Schmid 2003a). Here, the width of the Gaussian weight is ten times the boundary-layer thickness. It allows a very rich dynamical behaviour while ruling out disturbances that develop too far away from the attachment line, where the relevance of the swept Hiemenz flow model becomes questionable.

A spectral numerical scheme inspired by the one used by Obrist \& Schmid (2003b) to study the receptivity of the boundary layer has been implemented to solve the perturbation equations. It involves Chebyshev polynomials in the wallnormal direction and two Fourier transforms of the disturbances in the spanwise and chordwise directions. The latter requires periodic boundary conditions that are obtained by introducing a fringe region which smoothly drives disturbances to zero at $\pm x_{\max }$.

\subsection{Optimization}

The initial perturbation with an energy that is the most amplified over a finite time span $0<t<T$ is called the optimal perturbation. It maximizes the objective functional

$$
\mathscr{I}=\frac{E(T)}{E(0)} \text {. }
$$

A gradient algorithm described in Guégan et al. (2006) was used to determine the maxima of $\mathscr{I}$. The energy amplification of an arbitrary initial disturbance is iteratively improved by computing the gradient of the objective functional with respect to the initial disturbance.

In the present study the optimization algorithm is initialized with an initial disturbance in the shape of a random vorticity patch. After less than a dozen iterations, each additional iteration improves the objective functional by less than $10^{-4}$ times its current value, at which point the algorithm is considered to have converged. It should be mentioned that the algorithm consistently converges to the same solution when starting from a variety of initial guess values, which tends to show that a global maximum is reached independently of the initial guessed disturbance.

\section{Three-dimensional optimal disturbances}

\subsection{Transient energy growth}

Figure 2(a) displays the energy growth of the $k=0.25$ perturbations which yield optimal amplification at times $T=20,70,120$, for a Reynolds number $R e=550$. The envelope of all energy curves represents the maximum energy amplification that $k=0.25$ disturbances may experience at time $T$ at this Reynolds number. The growth is seen to be maximum at $T_{\max }=72$ where the energy of the optimal perturbations experiences a total gain of $G_{\max }=210$.

The envelopes of the energy curves for spanwise wavenumbers $k=0, k=0.25$ and $k=0.5$ are displayed in figure $2(b)$. The maximum energy growth $G_{\max }$ and the 

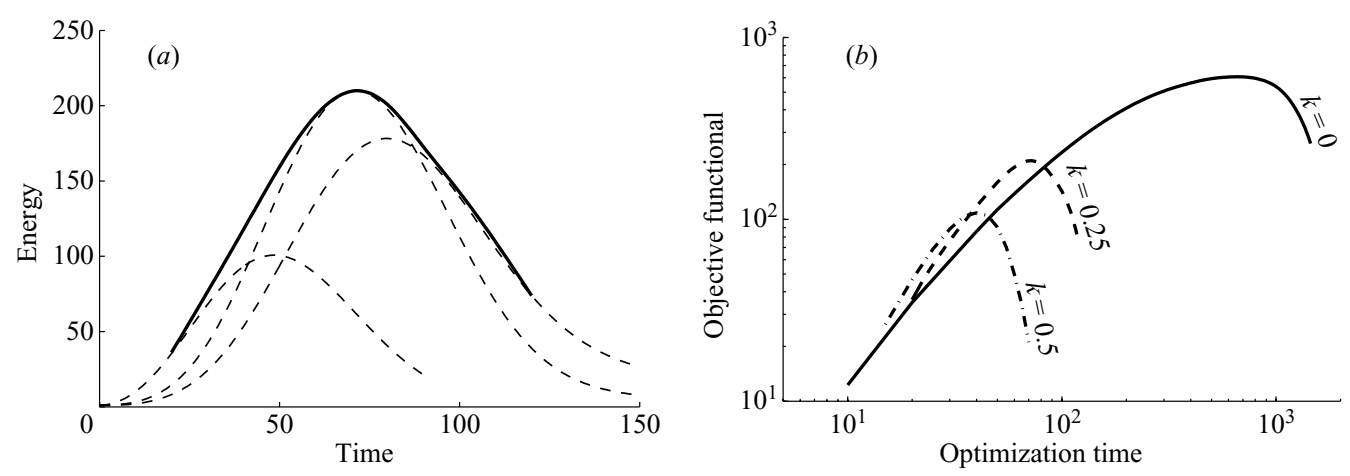

FIGURE 2. (a) Time evolution of the perturbation energy for $k=0.25$ optimal disturbances at times $T=20,70,120$ (dashed), at a Reynolds number $R e=550$. The envelope (solid) represents the maximum energy amplification that can be reached by $k=0.25$ disturbances in the time interval $20<t<120$. (b) Maximum energy amplification in the time interval $15<t<1500$ for perturbations at $k=0$ (solid), $k=0.25$ (dashed), $k=0.5$ (dash-dotted) and $R e=550$.
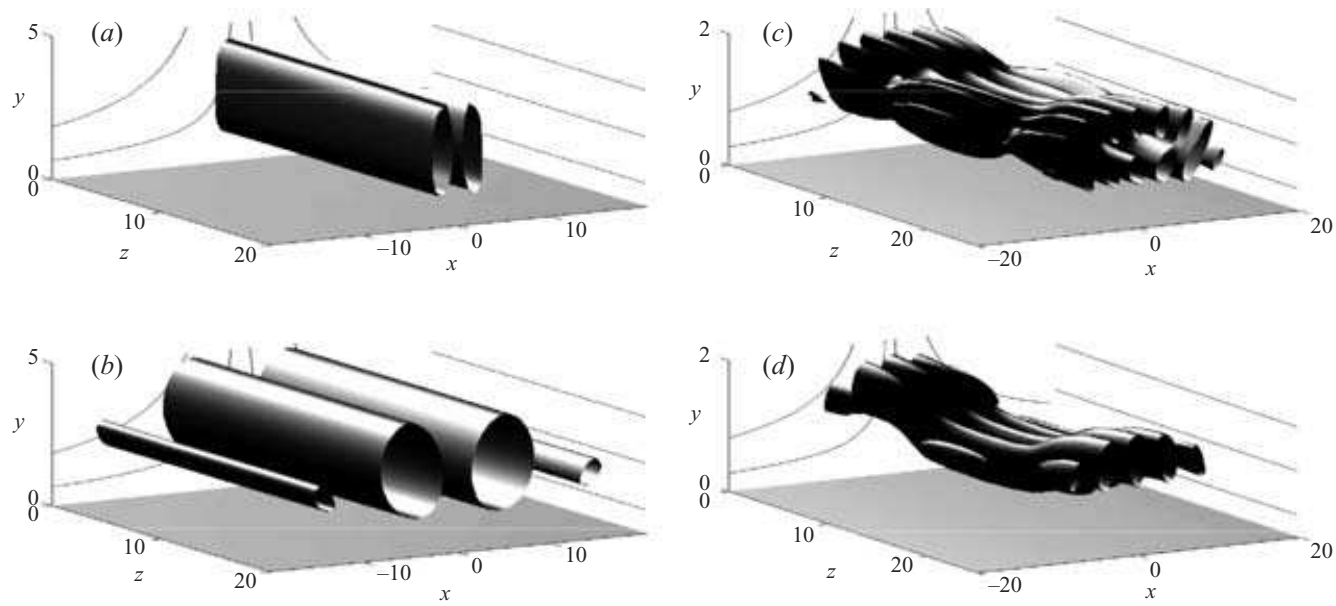

FIgURE 3. Homogeneous $k=0$ optimal perturbations at $(a) t=0$ and $(b)$ maximum amplification time $T=675 ;(c)$ and periodic $k=0.25$ optimal perturbations at $(d) t=0$ and maximum amplification time $T=72$, identified with the $Q$-criterion method. The isosurfaces are located at $20 \%$ of the maximum of $Q=\frac{1}{2}\left(\Omega_{i j} \Omega_{i j}-S_{i j} S_{i j}\right)$ where $\Omega_{i j}$ and $S_{i j}$ are the components of the vorticity and rate-of-strain tensor respectively. The Reynolds number is $R e=550$.

associated time $T_{\max }$ both decrease with wavenumber $k$. The maximum amplification is observed for homogeneous perturbations in the spanwise $z$-direction, in which case the energy growth is $G_{\max }=600$ at $T_{\max }=675$. It should be emphasized that perturbations at non-zero wavenumbers must not be discarded since they are more amplified than homogeneous ones on shorter time scales.

\subsection{Optimal perturbation structure}

The optimal homogeneous perturbation consists of spanwise vortices identified with the $Q$-criterion (Hunt, Wray \& Moin 1988) and displayed in figure 3(a). The initial disturbance consists almost exclusively of spanwise vorticity, as shown in figure 4(a). 
Through a mechanism similar to the lift-up mechanism (Landahl 1980) high-spanwisemomentum fluid is pushed toward the wall, and low-spanwise-momentum fluid is pulled away from the wall as shown in figure 5. Positive spanwise perturbation velocity $w$ is created where the jet between two spanwise vortices is directed toward the wall; outward jets generate negative $w$. As a consequence wall-normal $\omega_{y^{-}}$and chordwise $\omega_{x}$-vorticity components are amplified and dominate over the spanwise $\omega_{z}$ vorticity component at the time when the energy is maximum (figure $4 b$ ). Owing to the stretching induced by the base flow the disturbances initially localized about the flowdividing plane $x=0$ spread in the chordwise $x$-direction. This phenomenon is not observed in the paradigmatic lift-up configuration in a two-dimensional boundary layer, where the perturbation takes the shape of streamwise vortices periodically spaced in the transverse direction.

At finite $k$ the optimal disturbances take the shape of spanwise vortices meandering in the wall-normal direction as displayed in figure 3(c). At the maximum amplification time $T=72$ the vortices are wider and more intense (figure $3 d$ ). Isosurfaces of the vorticity components displayed in figure 6(a) reveal elongated structures initially tilted against the spanwise shear $W^{\prime}$ as in Hoepffner et al. (2005). The structures are also initially tilted in the direction of the weaker chordwise shear, which also seems to be the case in Hoepffner et al., but no explanation could be found for why this leads to optimal energy growth.

When the perturbation energy is maximal (figure 6b) the structures have flipped over and are tilted in the same direction as the spanwise shear but in the opposite direction to the chordwise shear. The chordwise vorticity component $\omega_{x}$ is the most amplified in this process.

\section{Discussion}

\subsection{Perturbation energy equation}

To further analyse the amplification mechanism one may extract the production and the dissipation terms from the energy equation. The time derivative of the total perturbation energy is

$\partial_{t} E=\int_{y>0} \lambda_{E}\left(-w v \partial_{y} W-u v \partial_{y} U+R e^{-1} \boldsymbol{u} \cdot \Delta \boldsymbol{u}-\boldsymbol{u} \cdot \boldsymbol{U} \nabla \boldsymbol{u}-\partial_{x} U u^{2}-\partial_{y} V v^{2}\right) \mathrm{d} x \mathrm{~d} y \mathrm{~d} z$.

The stretching direction $x$ and the sweep direction $z$ are associated with the production terms $-u v \partial_{y} U$ and $-w v \partial_{y} W$, displayed in figure $7(a)$ along with the dissipation term $R e^{-1} \boldsymbol{u} \cdot \Delta \boldsymbol{u}$ and the time derivative of the energy $\partial_{t} E$. The energy amplification may be mainly attributed to the spanwise production term $-w v \partial_{y} W$ balanced by the dissipation term $R e^{-1} \boldsymbol{u} \cdot \Delta \boldsymbol{u}$, while the chordwise production term $-u v \partial_{y} U$ and the other remaining terms are negligible.

A parallel can be drawn between the present analysis and the study of Hoepffner et al. (2005), who computed optimal perturbations of boundary-layer streaks. In both configurations the advection is stronger in a particular direction, respectively the direction of the streaks (referred to as the ' $x$ '-direction) or the direction of the sweep (denoted by $z$ throughout the present study), which thus defines a preferential downstream direction. As in the present case, Hoepffner et al. (2005) observe that the optimal perturbation with a finite streamwise wavenumber is initially tilted against the main shear and points downstream when its energy is maximum. The main 

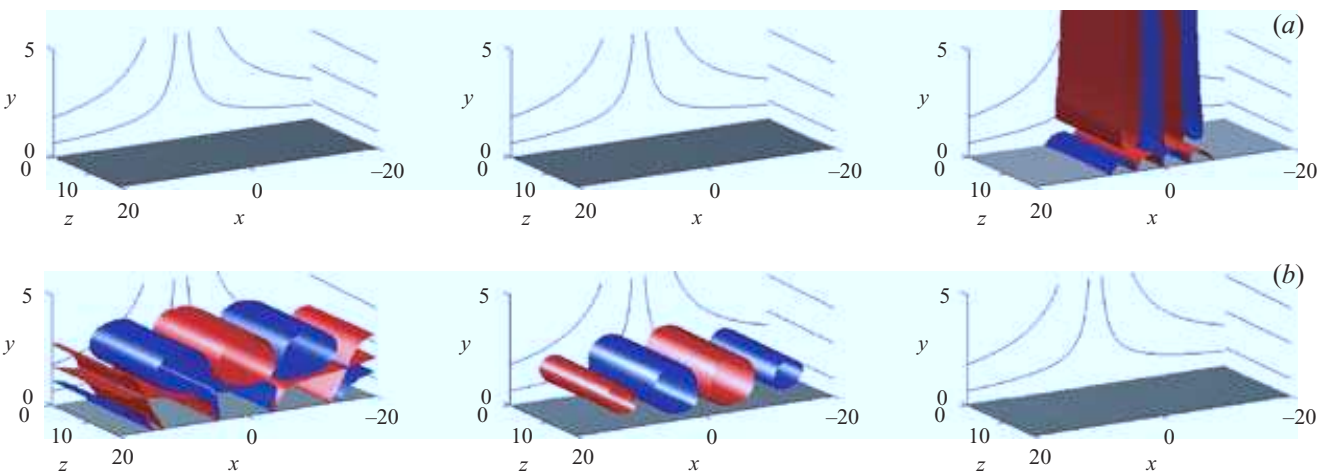

FIGURE 4. From left to right: chordwise, wall-normal and spanwise vorticity components of homogeneous $k=0$ optimal perturbations at $(a) t=0$ and $(b)$ maximum amplification time $T=675$. The isosurfaces correspond to 0.1 (red) and -0.1 (blue) times the maximum of the strongest vorticity component. At $t=0$ the vorticity maxima are $\omega_{x 0}=0, \omega_{y 0}=0.01, \omega_{z 0}=1$, and at $t=T, \omega_{x T}=23.8, \omega_{y T}=6.5, \omega_{z T}=0.7$. The Reynolds number is $\operatorname{Re}=550$.
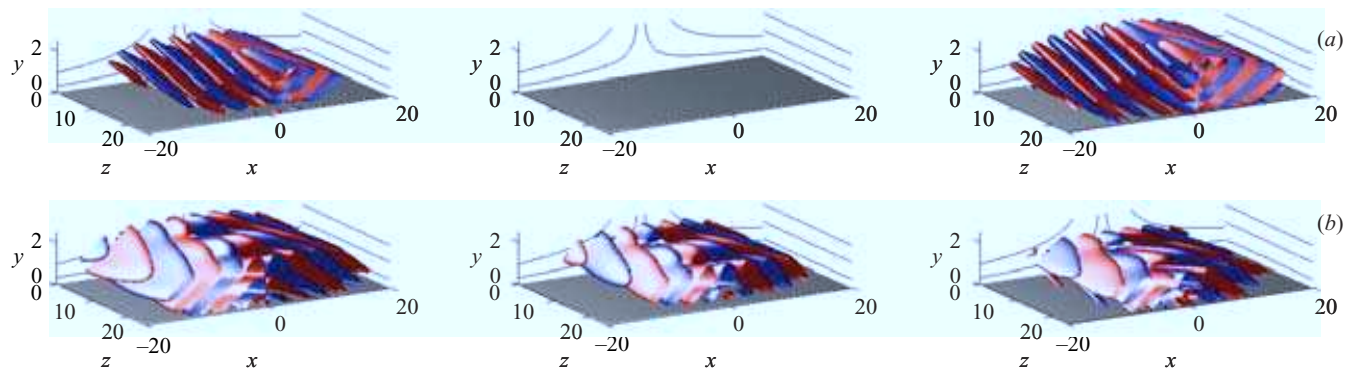

FIGURE 6. From left to right: chordwise, wall-normal and spanwise vorticity components of $k=0.25$ optimal perturbations at $(a) t=0$ and $(b)$ maximum amplification time $T=72$. The isosurfaces correspond to 0.1 (red) and -0.1 (blue) times the maximum of the strongest vorticity component. At $t=0$ the vorticity maxima are $\omega_{x 0}=0.31, \omega_{y 0}=0.01, \omega_{z 0}=1$, and at $t=T, \omega_{x T}=9.4, \omega_{y T}=3.9, \omega_{z T}=3.3$. The Reynolds number is $R e=550$.

contributor to the energy amplification in their study is their term ' $-u w \partial_{z} U$ '. The equivalent term here would be $-w u \partial_{x} W$ but since the sweep $W$ is homogeneous in the chordwise $x$-direction, it is equal to zero. The other production term ' $-u v \partial_{y} U$ ' in the streaky base flow contributes moderately to the energy growth but its counterpart $-w v \partial_{y} W$ in swept Hiemenz flow is responsible for most of the amplification. The chordwise production term $-u v \partial_{y} U$ has a counterpart ' $-w v \partial_{y} W$ ' which is zero in the boundary layer with streaks. Here it is negligible.

\subsection{Transient growth mechanisms}

Two distinct mechanisms are classically taken as responsible for energy amplification in shear flows, namely the Orr mechanism (Orr 1907) and the lift-up mechanism (Landahl 1980). In the Orr mechanism counter-rotating vortices parallel to the main stream vorticity and tilted against the mean shear are amplified by the basic shear; this mechanism is efficient at high streamwise wavenumbers on short time scales as demonstrated by Butler \& Farrell (1992). In the so-called lift-up mechanism streamwise vortices interact with the basic shear to generate streamwise perturbation velocity; this mechanism operates at smaller wavenumbers, on larger 


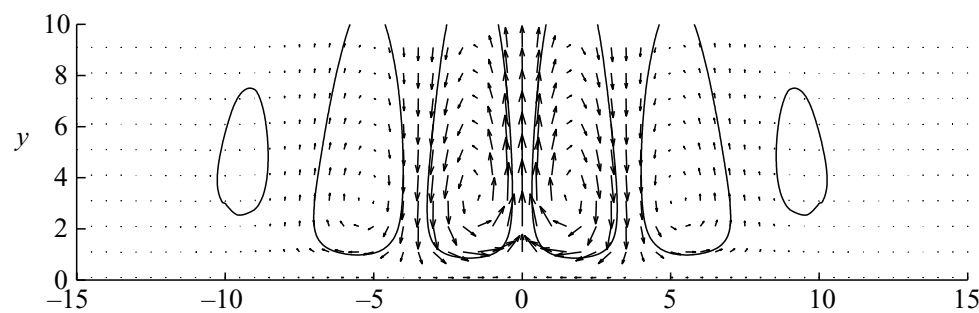

(a)
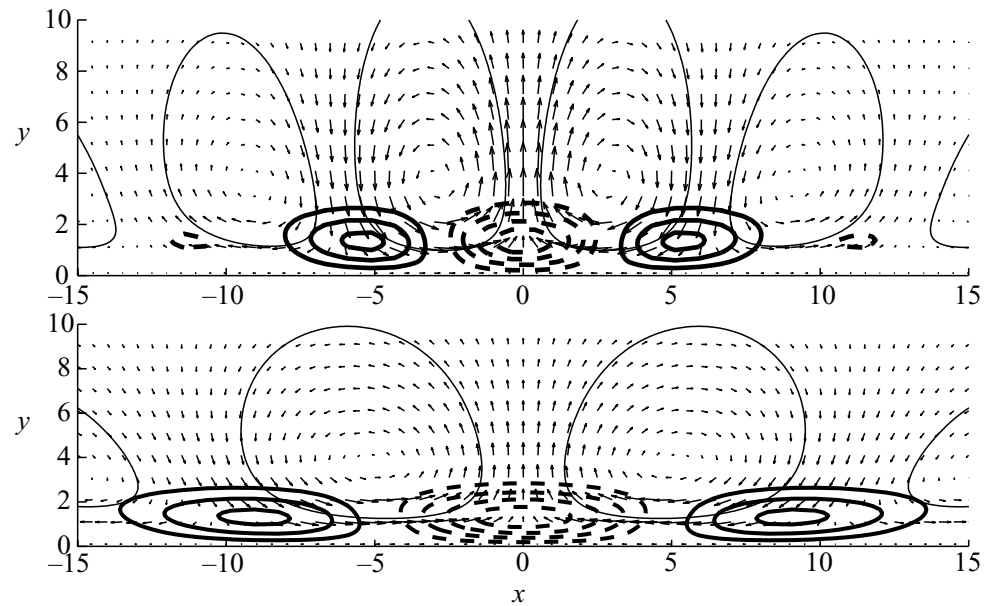

FIGURE 5. Vector plot of the chordwise and wall-normal $(u, v)$ velocity components in the $(x, y)$-plane and contours of spanwise perturbation velocity $w$ for a homogeneous $k=0$ optimal perturbation. The snapshots are taken at time $(a) t=0,(b) t=333$ and $(c)$ maximum amplification time $T=675$. Positive (negative) values of $w$ are displayed in thick solid (dashed) lines, and the contour levels and vector scale are the same in all three plots. To guide the eye, one streamline of each spanwise vortex is displayed as a thin solid line. The Reynolds number is $R e=550$.
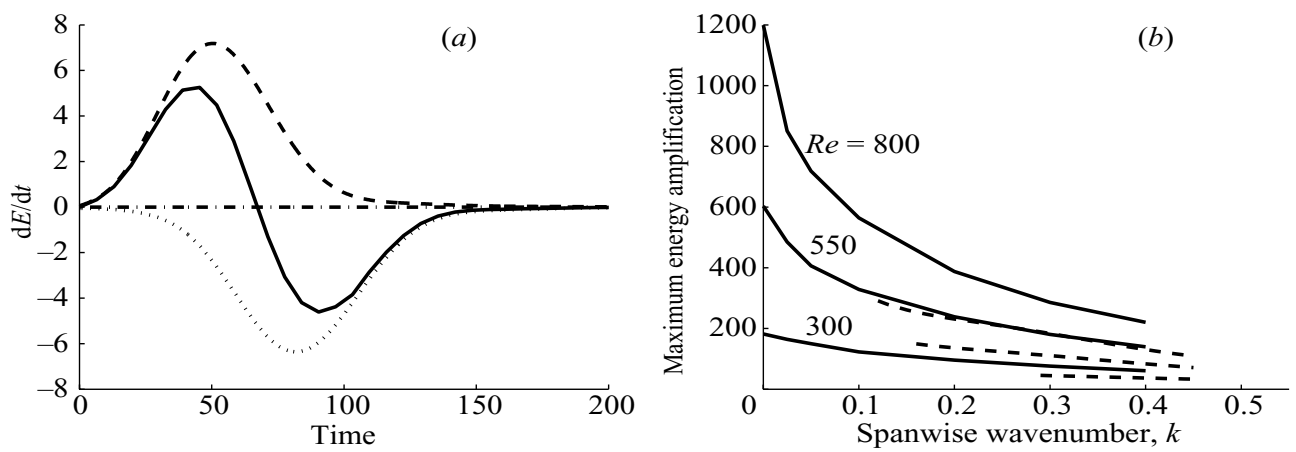

Figure 7. (a) Temporal evolution of the time derivative of the energy $\left(\partial_{t} E\right.$, solid), of the viscous dissipation $\left(R e^{-1} \boldsymbol{u} \cdot \Delta \boldsymbol{u}\right.$, dotted line) and of the two production terms $-u v \partial_{y} U$ (dash-dotted line) and $-w v \partial_{y} W$ (dashed line), for the optimal disturbance at $R e=550, k=0.25$, $T=70$. (b) Maximum energy growth of Görtler-Hämmerlin (dashed lines) and general optimal perturbations (solid lines) as a function of spanwise wavenumber $k$, for $R e=800,550,300$ (top to bottom).

time scales (Butler \& Farrell 1992). At medium wavenumbers both mechanisms work in conjunction and the optimal perturbation is a combination of streamwise and transverse vorticity. 

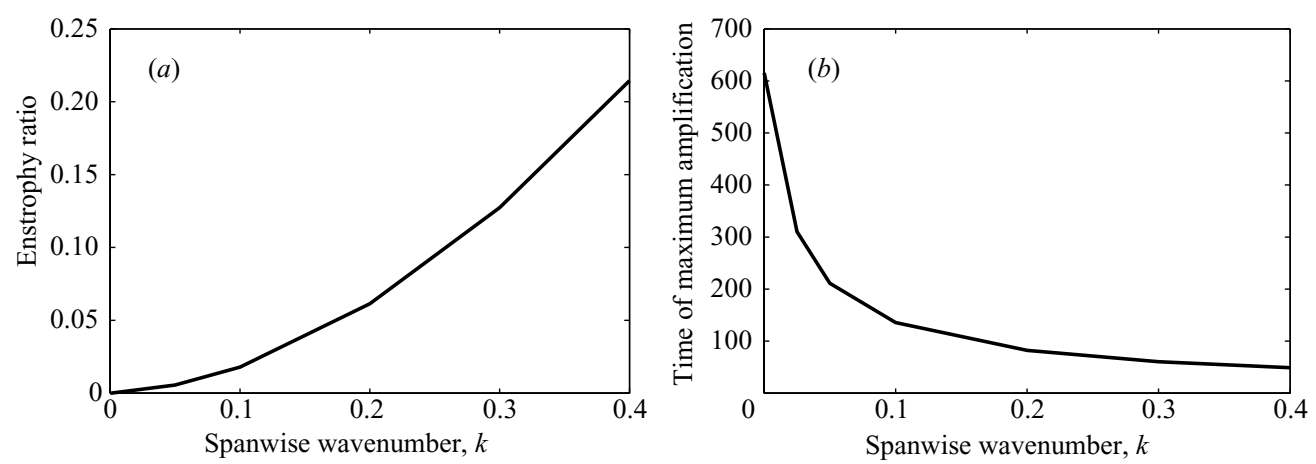

FIGURE 8. (a) Ratio of the initial enstrophy $\int_{X Y Z} \omega_{x}^{2}(t=0) \mathrm{d} x \mathrm{~d} y \mathrm{~d} z$ associated with vorticity in the chordwise $x$-direction, and initial enstrophy $\int_{X Y Z} \omega_{z}^{2}(t=0) \mathrm{d} x \mathrm{~d} y \mathrm{~d} z$ associated with vorticity in the spanwise $z$-direction, versus spanwise wavenumber $k$. The chordwise vorticity component of the optimal perturbation increases with $k$ compared to the spanwise component and the maximum amplification time decreases with $k$. (b) Optimal perturbation amplification time versus $k$. It is maximum at $k=0$. The Reynolds number is $R e=550$.

Figure $8(a)$ displays the ratio of the initial chordwise enstrophy $\int_{X Y Z} \omega_{x}^{2}(t=0) \mathrm{d} x \mathrm{~d} y \mathrm{~d} z$ and the initial spanwise enstrophy $\int_{X Y Z} \omega_{z}^{2}(t=0) \mathrm{d} x \mathrm{~d} y \mathrm{~d} z$ for optimal disturbances at different spanwise wavenumbers $k$, and $R e=550$. This ratio gives a complete picture of the balance between vorticity components in the initial disturbance since the wall-normal vorticity component accounts for less than $1 \%$ of the total enstrophy at all wavenumbers investigated. At zero spanwise wavenumber the chordwise $x$-enstrophy is negligible compared to the spanwise $z$-enstrophy, and the optimal perturbation consists of spanwise vortices, in line with the results of Butler \& Farrell (1992). As the spanwise wavenumber $k$ is increased the chordwise $\omega_{x}$-component accounts for an increasing fraction of the initial enstrophy; at $k=0.4$ the chordwise $x$-enstrophy is a fifth of the spanwise $z$-enstrophy.

Chordwise vorticity amplification via an Orr-like mechanism clearly becomes more dominant on lift-up as $k$ is increased. Consistently (Butler \& Farrell 1992), the maximum amplification time dramatically decreases as $k$ is increased from $k=0$ to $k=0.4$ (figure $8 b$ ).

\subsection{Comparison with Görtler-Hämmerlin disturbances}

Optimal Görtler-Hämmerlin perturbations consist of chordwise vortices amplified by a two-dimensional mechanism reminiscent of the Orr mechanism (Guégan et al. 2006). At high spanwise wavenumbers $k$ general optimal disturbances are amplified by the same type of mechanism. The amplification levels displayed in figure $7(b)$ are of the same order of magnitude as those of Görtler-Hämmerlin optimal perturbations for $k>0.1$, and the optimal energy amplification has a similar $k$-dependence. Note that a strict quantitative comparison cannot be made since amplification in the GörtlerHämmerlin case is defined with a top-hat weight function in the chordwise direction (Guégan et al 2006).

Chordwise vorticity contours in $(z, y)$-planes (figure $9 b)$ display similar features to optimal Görtler-Hämmerlin disturbances (figure $9 a$ ), although on shorter time scales. In the latter case an array of counter-rotating chordwise vortices initially bent against the spanwise shear eventually tilts in the direction of the shear. Similarly in the general case, optimal perturbations are initially bent against the spanwise shear (figure $9 b, t=0$ snapshot) and then tilt in the direction of the shear (figure $9 b, t=70$ 
(a)
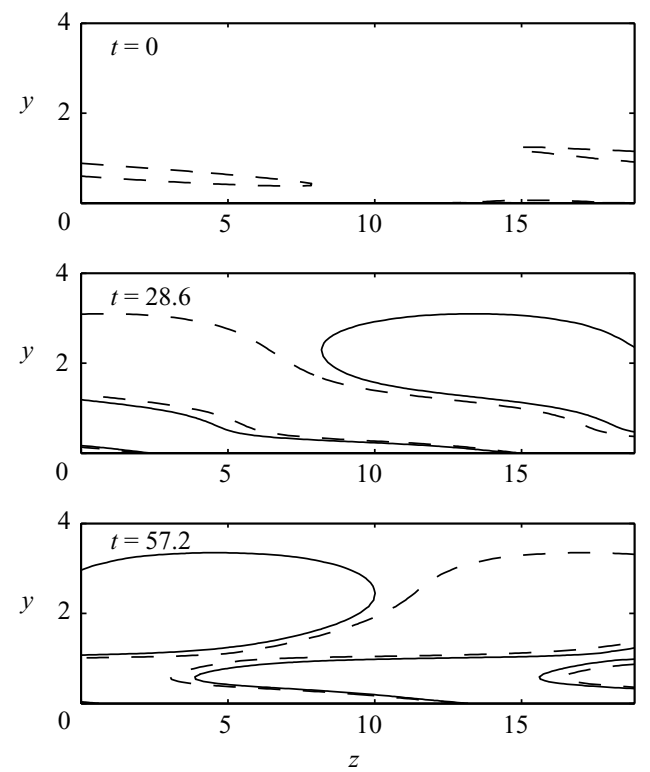

(b)
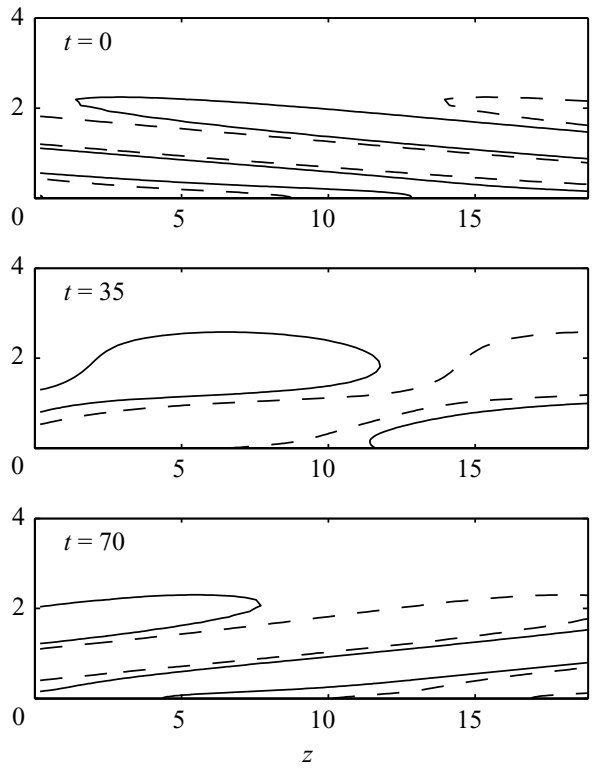

FIGURE 9. Chordwise vorticity contours in the $(z, y)$-plane of $(a)$ an optimal GörtlerHämmerlin perturbation and $(b)$ a general optimal perturbation at $k=0.25$ and $R e=550$. The successive snapshots were taken (from top to bottom) at times $t=0, t=T / 2$ and $t=T$. For easier visualization the levels have been set at $2 \%$ and $20 \%$ of the maximum chordwise vorticity in $(a)$ and $(b)$ respectively.

snapshot). For intermediate times (figure $9 b, t=35$ snapshot) the occasional vortex splitting observed by Guégan et al. (2006) is recovered as well.

The Görtler-Hämmerlin assumption has been extensively used in the past, and it is here demonstrated to yield realistic energy amplification levels for spanwise wavenumbers $k>0.1$ and short time scales. The optimal mechanism at finite $k$ consists partly of amplification of the chordwise vorticity by the main shear $W^{\prime}$ in a similar way to optimal Görtler-Hämmerlin perturbations. However, in order to obtain the true shape of the optimal perturbations, it is necessary to relax the Görtler-Hämmerlin assumption: the optimal perturbations take the shape of spanwise vortices (figure $3 a$ ) whereas their Görtler-Hämmerlin counterparts consist of chordwise vortices. For similar reasons, the lift-up mechanism and the high amplification levels at $k=0$ can only be observed if the Görtler-Hämmerlin assumption has been relaxed.

The authors would like to thank C. Cossu for discussions on transient growth mechanisms. Alan Guégan holds a PhD fellowship from CNRS and the French "Délégation Générale pour l'Armement"; this study has also been supported by Airbus.

\section{REFERENCES}

Butler, K. M. \& Farrell, B. F. 1992 Three-dimensional optimal perturbations in viscous shear flow. Phys. Fluids A 4, 1637-1650.

Corbett, P. \& Bottaro, A. 2001 Optimal linear growth in swept boundary layers. J. Fluid Mech. 435, $1-23$. 
Gúgan, A., Schmid, P. J. \& Huerre, P. 2006 Optimal energy growth and optimal control in swept Hiemenz flow J. Fluid Mech. 566, 11-45.

HoepfFner, J., Brandt, L. \& Henningson, D. S. 2005 Transient growth on boundary layer streaks J. Fluid Mech. 537, 91-100.

Hunt, J. C. R., Wray, A. A. \& Moin, P. 1988 Eddies, streams, and convergence zones in turbulent flows. In Studying Turbulence Using Numerical Simulation Databases, 2. Proc. of the 1988 Summer Program, pp. 193-208.

LANDAHL, M. T. 1980 A note on an algebraic instability of inviscid parallel shear flows. J. Fluid Mech. 98, 243-251.

ObRist, D. \& SCHMid, P.J. $2003 a$ On the linear stability of swept attachment-line boundary layer flow. Part 1. Spectrum and asymptotic behavior. J. Fluid Mech. 493, 1-29.

Obrist, D. \& Schmid, P. J. $2003 b$ On the linear stability of swept attachment-line boundary layer flow. Part 2. Non-modal effects and receptivity. J. Fluid Mech. 493, 31-58.

OrR, W. M. F. 1907 The stability or instability of the steady motions of a liquid. Proc. R. Irish Acad. A 27, 9-69. 